\title{
Input Mezzanine Card for the Fast Tracker at ATLAS
}

\author{
Tomoya Iizawa on behalf of the ATLAS Collaboration
}

\begin{abstract}
The Fast Tracker is an integral part of triggev6 upgrade program for the ATLAS experiment. At LHC Run $2_{37}$ which started operations in June 2015 at a center of mass energy of $13 \mathrm{TeV}$, the luminosity could reach up to $2 \cdot 10^{34} \mathrm{~cm}^{-2} \mathrm{~s}^{-1}$ and an average of 40-50 simultaneous proton collisions per beam crossing will be expected. The higher luminosity demands $4^{0}$ more sophisticated trigger system with increased use of tracking information. The FTK is a highly-parallel hardware system that rapidly finds and reconstructs tracks in the ATLAS innerdetector at the triggering stage. This paper focuses on the Mezzanine Board that is input module of the entire FTK system.

The functions of this board are: receive the pixel and micro strip data from the ATLAS Silicon read-out drivers, performo clustering, and forward the data to its mother board. Mas\$ production and quality control tests of Mezzanine Boards were completed, and staged installation and commissioning are ongoing. Details of its functionality, mass production, quality control tests, and installation are reported.
\end{abstract}

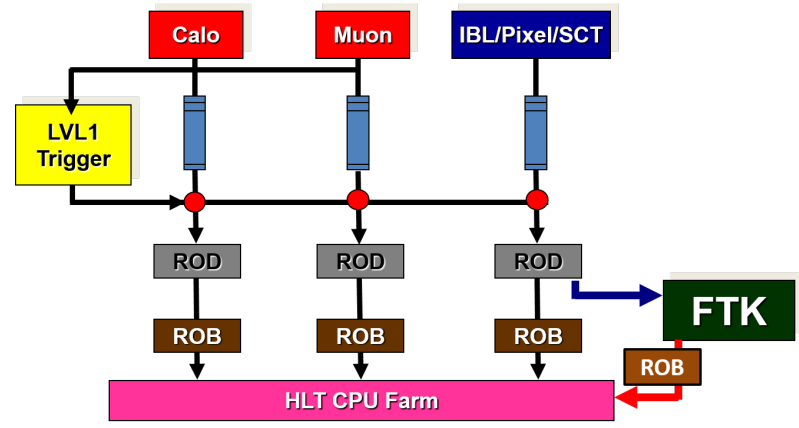

Fig. 1. The overview of the ATLAS trigger system with FTK.
The Fast TracKer (FTK) [2] is one of the ATLAS upgrade programs to keep physics sensitivity in the high pile-ups environment. It is an electronics system that will do global track reconstruction after each Level-1 trigger to enable the HLT to have early access to tracking information. FTK will use data from Insertable B-Layer (IBL) [3], pixel, and semiconductor tracker (SCT) detectors. The FTK performs track reconstructions in hardware with a high degree of parallelism allowing for the tracks to be readily available at the HLT.

This paper focuses on the FTK Input Mezzanine cards (IM) that are the input interface and the first processing stage of the FTK system.

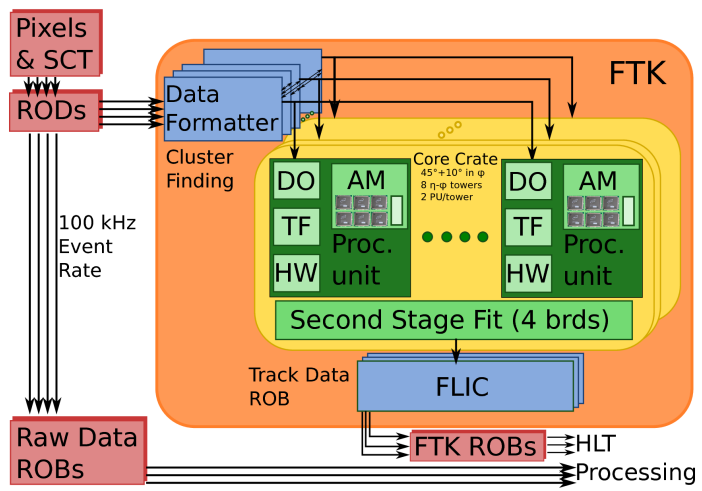

Fig. 2. Block diagram of the full FTK system.

\section{Functionality OF IM}

The IM functions are implemented in a mezzanine card of 12 layers with a size of $149 \mathrm{~mm} \times 74 \mathrm{~mm}$ that connects the DataFormatter (DF) [4] motherboard with a High Pin Count FPGA Mezzanine Card (FMC) connector. The IMs receive data from IBL, pixel, and SCT data with about 100 million channels. The cross-section view of the tracking detectors is shown in Figure. 3. Each IM receives data by up to 4 SLINK optical fibers from RODs through four SFP+ connectors. There are two types of IM, one uses Spartan-6 FPGA (XC6SLX150T) for processing pixel and SCT data, and the other uses Artix-7 FPGA (XC7A200T) for IBL/pixel and SCT data. The IMs with Spartan-6 and Artix-7 are shown in Figures. 4, 5, respectively. On each mezzanine there are 2 FPGAs. Each FPGA receives two links, one from IBL/pixel and the other from SCT, processes data independently, and transmits the output to the FMC connector. In each FPGA, a clustering algorithm [5] is implemented to reduce the volume of the input data as well as to improve precision of hit spacial 
measurements by identifying clusters. There are two types of clustering algorithms: 2D for IBL and pixel detectors and $1 \mathrm{D}$ for SCT strips. The later is aided by pre-clustering in the SCT Front End (FE) electronics. For the data transfers from IM to DF, Double Data Rate (DDR) source-synchronous parallel bus of LVDS operated at $200 \mathrm{MHz}$ is used. The Inter 90 Integrated Circuit (I2C) bus is used for slow control of IM from DF. Each FPGA is equipped with a $18 \mathrm{Mb}$ externad SRAM and a $32 \mathrm{Mb}$ flash memory. The IM is powered frons the FMC connector or an external power connector used for f $_{4}$ the standalone tests where there is not DF. The JTAG chain for FPGA configuration is accessible both from the $\mathrm{FMG}_{6}$ connector and an external connector. The S-LINK runs up tog 3.1 Gbps.

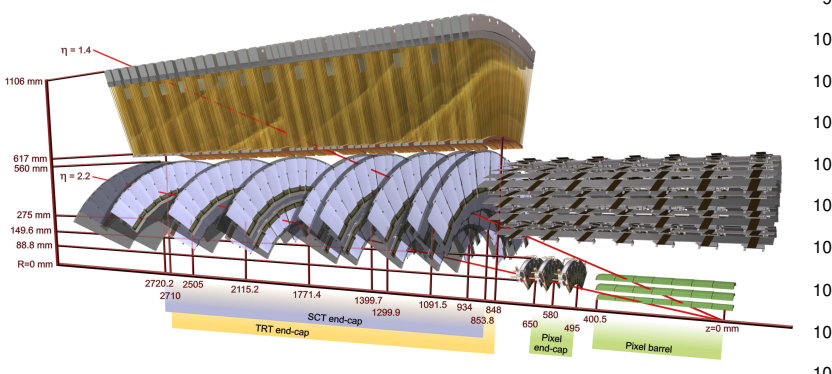

Fig. 3. The cross-section view of the ATLAS Inner Detector modules. The FTK uses the inner ones, Pixels and SCT.

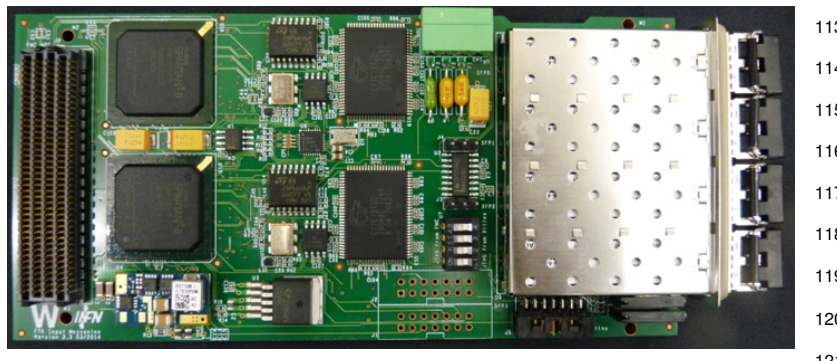

Fig. 4. The photograph of IM card. Two Spartan-6 FPGAs are implemented

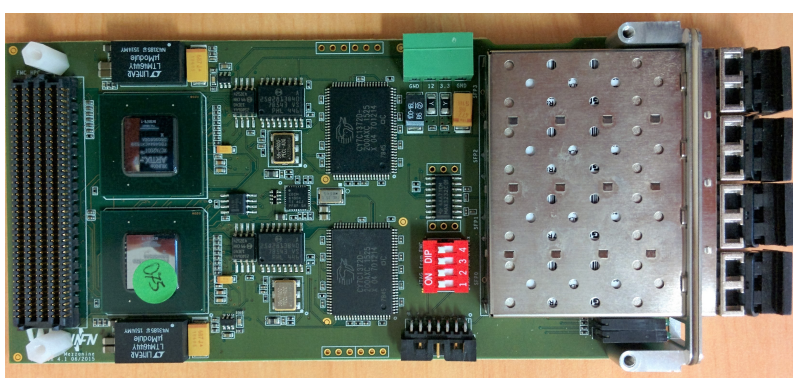

Fig. 5. The photograph of IM card. Two Artix-7 FPGAs are implemented.

\section{A. Detailed description of the clustering algorithm}

Clustering has two purposes, one is to reduce the volumes of the received data before further processing, and the others is to determine the cluster center for obtaining the besto spatial measurement. The clustering is implemented by using a 2D-clustering algorithm for the IBL and pixel detectors, while it is 1D-clustering for SCT.

For SCT data, 1D-clustering is performed, which is partly implemented at the SCT Front-End chips. Continuous strips are found and clustered, then cluster size and cluster center information are passed to DF.

A multi-core FPGA-based 2D-clustering algorithm is used for IBL and pixel detectors. The challenge is to perform $2 \mathrm{D}$ cluster finding in real time. The algorithm utilizes a sliding window technique with adjustable window size in order to minimize the FPGA resources required for cluster identification. A key element of this algorithm is the possibility to instantiate multiple clustering cores working on different windows that can be used in parallel to increase performance exploiting better the FPGA resources. In addition to the parallelization, the algorithm is executed in a pipeline, thus allowing for data preparation, clustering and cluster post processing to be executed in parallel.

The incoming data is transformed from the native detector format to a format which is useful to the following processing step. It is a pre-processing step that selects, formats and organizes the information that is used by the clustering algorithm such as start/end event words, module headers/trailers, and pixel hit words. Along with the format transformation, the incoming data is realigned. The ATLAS pixel modules 16 front-end (FE) chips are arranged in 2 rows, each of 8 readout chips, and they are numbered anticlockwise. The hit data are readout in the same FE sequence. This means that half of the pixel module data arrive in reverse column order than the other half. The hit decoder module needs to restore the order of the hits since the clustering algorithm is based on the assumption that hits are ordered by increasing column number sequence.

The finder logic starts with the first received hit. It defines the cluster window (21times 8 pixel size) with respect to the first hit position. Then the logic loads all hits within the window. Once all the hit data are loaded, the algorithm selects the first reference hit. Then on each clock cycle the hits that are in direct neighborhood with the selected hit are also selected until no directly neighboring hits remain. Eventually the selected hits are sent to the centroid calculator as a cluster.

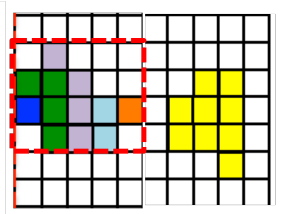

First hit used as seed for the clustering Hits selected in the second clock cycle Hits selected in the third clock cycle Hits selected in the forth clock cycle Hits selected in the fifth clock cycle

Hits belonging to a different cluster

Fig. 6. An illustration of the pixels and the window for the $2 \mathrm{D}$ clustering. Here $4 \times 5$ size window is shown as an example.

The cluster post-processing performs further data volume reduction and improve precision by calculating the cluster centroid. One fundamental characteristic of the 2D clustering 


\section{Production And test Status of IM}

The final version of the IMs were produced, and quality control tests were performed. The tests include visual checks, electrical checks, and bit-error-rate (BER) measurements. The BER of all IMs satisfies the ATLAS experiment requirements of $10^{-15}$. All produced IMs passed the tests, including enough spares. The mass produced IMs with Spartan-6 are shown in Figure. 7.

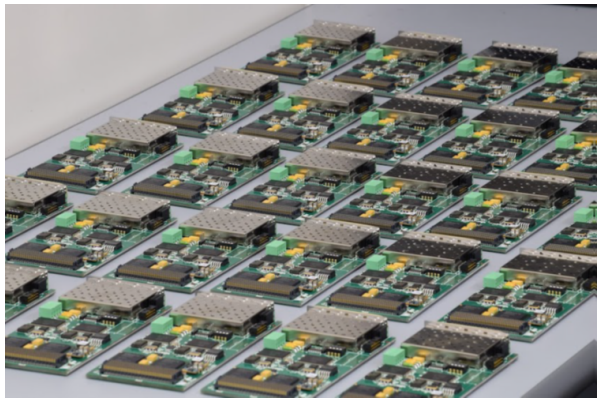

Fig. 7. A photograph of the mass produced IM cards.

implementation is that different clustering engine can work independently and in parallel to identify different clusters, therefore increasing performance while exploiting more FPGA resources.

Installation and integration tests are ongoing. Communicas tion with inner-detector RODs are confirmed for IBL, pixel and SCT. The DDR data transfer between DF is running at design level of $200 \mathrm{MHz}$. With 4 IMs mounted on $1 \mathrm{DF}$ board, stable dataflow is achieved at $100 \mathrm{kHz}$ event rate using full 16 input channels, with the clustering functionality working. For configuration and monitoring, I2C bus is fully tested and in use. Several registers for monitoring are defined and read out via I2C. More will be added as necessary. The output of IM hardware was compared with that of the emulation, and was found to match exactly the simulation. Now IMs are being installed, and tested under real operation conditions. The installed IMs and DFs are shown in Figure. 8. Data taking with SCT detector was established in 2015 with input event rate of up to $100 \mathrm{kHz}$. For real IBL/pixels, firmware development is ongoing to cope with special data.

The "First" track of FTK will come soon, and FTK will start operation/commissioning by the start of the physics run in 2017 .

\section{Conclusions}

In this paper the functionality and current development status of the IM are described. The IM is the input stage of the entire FTK system. The mass production of IMs was completed, integration tests and staged installation are ongoing.

FTK will start data taking with full coverage $(|\eta|<2.5)$ in the early 2017 , and will be upgraded.

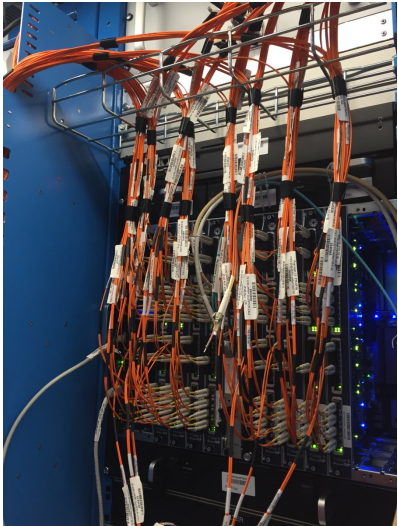

Fig. 8. IMs and DFs are being installed in the ATLAS DAQ.

\section{REFERENCES}

[1] ATLAS Collaboration, The ATLAS Experiment at the CERN Large Hadron Collider, 2008 JINST 3 S08003.

[2] ATLAS Collaboration, Fast TracKer Technical Design Report, https://cds.cern.ch/record/1552953/files/ATLAS-TDR-021.pdf, 2013.

[3] ATLAS collaboration, ATLAS Insertable B-Layer technical design report, CERN-LHCC-2010-013, 2010.

[4] Y.Okumura, J.Olsen, T.Liu, Prototype performance studies of a Full Mesh ATCA-based General Purpose Data Processing Board, arXiv:1403.4331v1, 2014.

[5] C.L. Sotiropoulou, S. Gkaitatzis, A. Annovi, M. Beretta, K. Kordas, S. Nikolaidis, C. Petridou, G. Volpi, A parallel FPGA implementation for real-time 2D pixel clustering for the ATLAS Fast Tracker Processor, JINST 9 C10018, 2014.

[6] Y. Nakahama, The ATLAS Trigger System: Ready for Run-2, Journal of Physics: Conference Series 664 (2015) 082037 